\title{
An Improved Method to Determine the Antenna Factor
}

\author{
Wout Joseph and Luc Martens, Member, IEEE
}

\begin{abstract}
In this paper, we present an improved method to determine the antenna factor of three antennas. Instead of using a reflecting ground plane we use absorbers. Destructive interference between the direct beam and the residual reflected beam from the absorbers is avoided by splitting the measured frequency range in bands and changing the distance between the two antennas depending on the frequency band. Furthermore, this method is applicable for both E- and H-field probes. Our method has also the advantage of being low-cost: The method does not need to be performed in an anechoic chamber to obtain high accuracy. To take the residual reflections of the environment into account, we perform a de-embedding procedure. We have developed two de-embedding methods.
\end{abstract}

Index Terms-Antenna factor, calibration, dipole antenna, splitshield loop antenna.

\section{INTRODUCTION}

D URING THE last decade, the use of mobile telephones [especially global system for mobile communications (GSM) phones] has increased enormously. At the same time, the question whether the fields of the base stations are harmful, becomes more and more important. So there is a need to measure electromagnetic fields at frequencies around 900 (GSM900) and 1800 (GSM1800) MHz. Electromagnetic fields are also measured around sources for use in electromagnetic compatibility studies. Accurate measuring of electromagnetic fields can only be done if the electromagnetic-field probes are calibrated carefully. Antenna calibration involves the determination of the antenna factor (AF). The antenna factor is defined as follows:

$$
\mathrm{AF}=20 \cdot \log \left(\frac{E}{V}\right) \quad\left[\mathrm{dB}\left(\mathrm{m}^{-1}\right)\right]
$$

where $E$ is the incident electric field at the antenna to be calibrated and $V$ is the voltage measured at the terminals of the antenna to be calibrated.

The principle of using three antennas for the calibration is introduced by Smith [1], [2]. In this method, the reflected beam is taken into account theoretically. Reflections can largely disturb the measurements. The theoretical site attenuation values in the ANSI C63.4 [3] are based on [1] and [2]. Antenna measurements on different types of open-area test sites (OATS) with different conducting planes are reported in [4]. In this paper, an absorbing ground plane is used to reduce the reflections. Several improvements to the method of Smith have been published

Manuscript received April 24, 2002; revised March 15, 2004

The authors are with the Department of Information Technology, Ghent University, B-9000 Ghent, Belgium (e-mail: wout.joseph@intecUGent.be).

Digital Object Identifier 10.1109/TIM.2004.838116
[5] and [6]. These methods make use of tabulated values of the maximum field strength for frequencies below $1000 \mathrm{MHz}$. An advantage of our method is that it is applicable for both Eand $\mathrm{H}$-field probes. For the calibration of loop antennas, two methods are described in [7]. The first method is based on calculation of the loop impedances. The second method is by generating a well-defined standard magnetic field. The first method cannot be used because the geometric shape of the split-shield loop probes is not simple. For the second method, the generation of a well-defined standard magnetic field is necessary. Our method does not need such a standard magnetic field. The method of Glimm et al. [8] is only applicable for directional antennas and not for loop antennas.

Mostly, measurements to determine the antenna factor are done in an anechoic chamber. However, this is expensive. If one wants to optimize the gain and the sensitivity of the measurement antennas, several characterization cycles are needed. Therefore, the use of a low-cost method is advised.

In this paper, we present a method based on S-parameter measurements. The method does not make use of tabulated values as in [5] and [6]. The frequency range that we investigate is from 600 to $2000 \mathrm{MHz}$. This range contains the GSM900 and GSM1800 frequencies. With our method, the antenna calibration must not be performed in an anechoic chamber but is done in an indoor open-site surrounded by absorbers to minimize interference. This makes it a low-cost method delivering accurate results.

A limitation to our method is that it is only applicable for pairs of omnidirectional antennas and when the antennas are carefully aligned. By using appropriate stands, this alignment can be done properly.

In Section II, our method is explained. The results are described in Section III. The conclusions are presented in Section IV.

\section{METHOD}

\section{A. Configuration}

The calibration setup is shown in Fig. 1. We use a well-absorbing material on the ground and name this an absorbing ground plane.

We make sure that the far-field conditions are fulfilled

$$
R \geq \frac{2 D^{2}}{\lambda}
$$

with $R$ the distance from the antenna, $D$ the maximum dimension of the antenna, and $\lambda$ the wavelength at the operation frequency. 


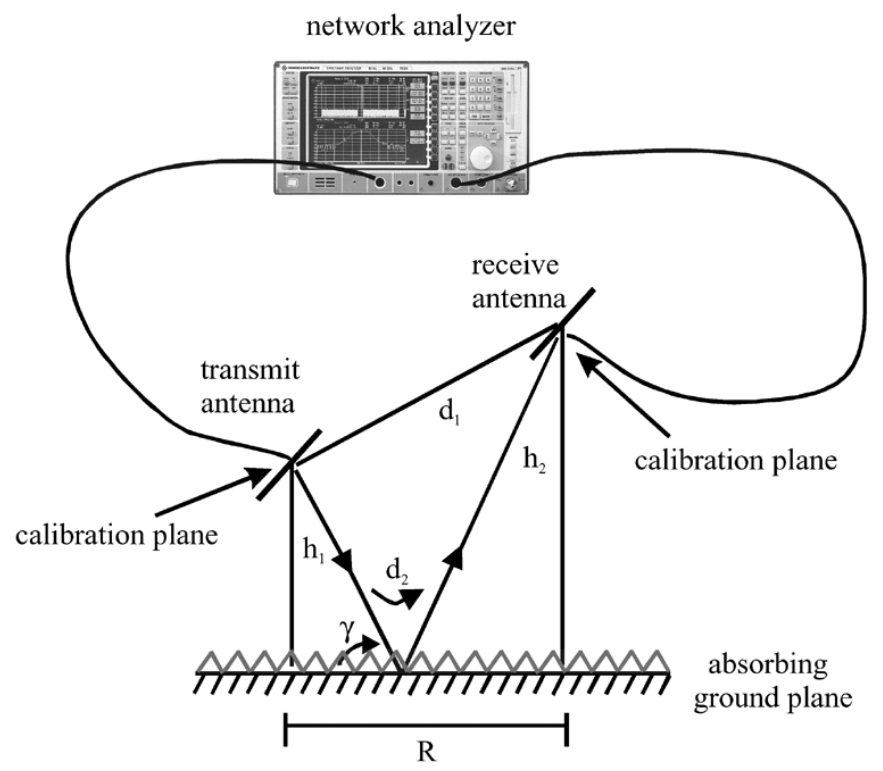

Fig. 1. Measurement setup.

Measurements are made with a network analyzer (Rohde and Schwarz ZVR). The network analyzer is calibrated with a SOLT calibration, using the standards short, open, load, and thru. By connecting these standards with the coaxial connectors of the measurement planes and using the SOLT-calibration formulas, the reference planes of the measurements are moved to the inputs of the antennas as indicated on Fig. 1. The antenna factors are determined between 600 and $2000 \mathrm{MHz}$.

The antennas used in the method are dipoles with lengths $15 \mathrm{~cm}(\lambda / 2$ at $900 \mathrm{MHz})$ and $7.5 \mathrm{~cm}(\lambda / 2$ at $1800 \mathrm{MHz})$, a conical dipole antenna and an in-house made split-shield loop antenna, one terminated in a $50 \Omega$ load and the other one in a $0 \Omega$ load, shown in Fig. 2. The split-shield loop antenna is designed to reject the contribution of the electric field to the magnetic-field measurement [9]. Both split-shield loop antennas have a diameter of $3.4 \mathrm{~cm}$.

\section{B. Theory of the Method}

We start from the Friis formula [10], [11]

$$
\mathrm{P}_{\mathrm{r}}=\mathrm{P}_{\mathrm{i}} \mathrm{G}_{\mathrm{t}} \mathrm{G}_{\mathrm{r}}\left(\frac{\lambda}{4 \pi \mathrm{d}_{1}}\right)^{2} \cdot \mathrm{PLF}
$$

where $P_{r}$ is the received power delivered to the receiver load, $P_{i}$ is the available input power of the transmitting antenna, $G_{r}$ is the realized (apparent) gain of the receiving antenna, and $G_{t}$ is the realized gain of the transmitting antenna. $G_{r}$ and $G_{t}$ contain the mismatch of the receiving and the transmitting antenna. Figs. 3(a) and (b) clarify the notations used in (3).

PLF stands for polarization loss factor. It is defined as PLF = $\left|\overline{\mathrm{e}}_{\mathrm{W}} \cdot \overline{\mathrm{e}}_{\mathrm{a}}\right|^{2}=|\cos \psi|^{2}$ where $\overline{\mathrm{e}}_{\mathrm{W}}$ is the unit vector of the incident field, $\bar{e}_{\mathrm{a}}$ is the unit vector of the polarization of the field of the receiving antenna, and $\psi$ the angle between the two unit vectors. $\mathrm{d}_{1}$ is the distance between both antennas.

If we measure the $\mathrm{S}_{21}$-parameter (with respect to $50 \Omega$ ) between the calibration planes of the antennas, the attenuation is

$$
\mathrm{A}=\frac{\mathrm{P}_{\mathrm{r}}}{\mathrm{P}_{\mathrm{i}}}=\left|\mathrm{S}_{21}\right|^{2} \text {. }
$$

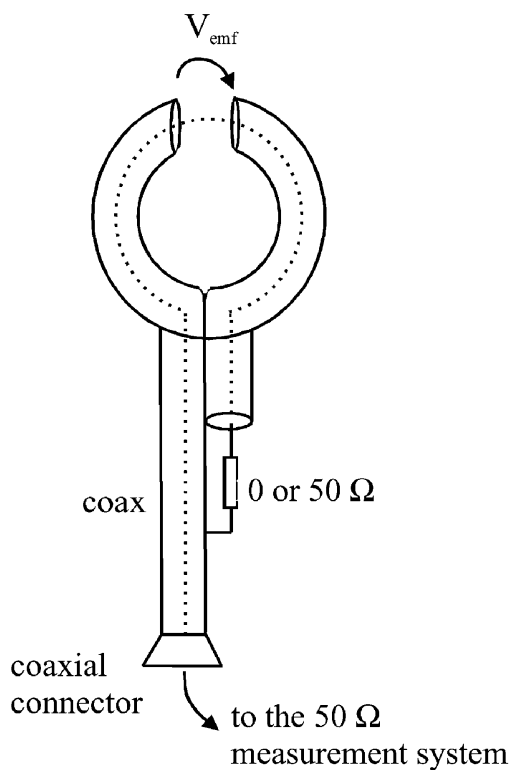

Fig. 2. Geometry of the split-shield loop antenna with loading resistance of 0 or $50 \Omega$.
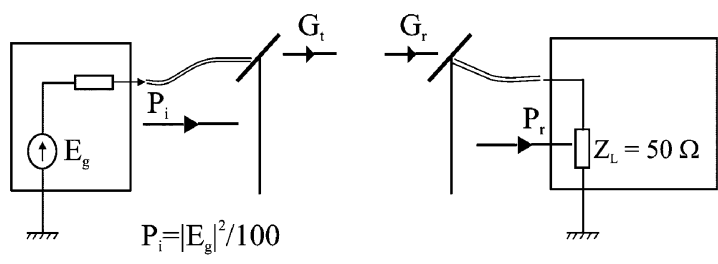

(a)

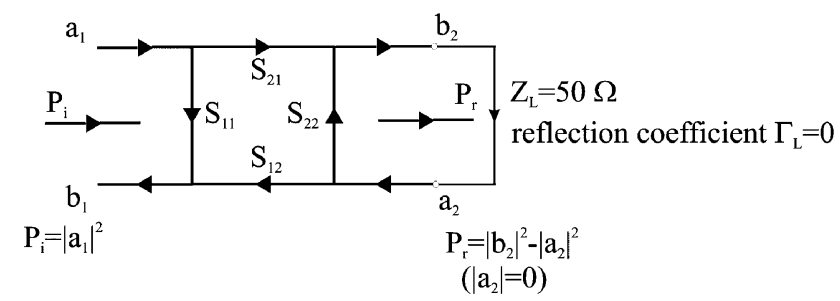

(b)

Fig. 3. (a) Antenna setup with indication of powers and (b) its S-parameter equivalent.

Combining (3) and (4), we obtain

$$
\left|\mathrm{S}_{21}\right|^{2}=\mathrm{G}_{\mathrm{t}} \mathrm{G}_{\mathrm{r}}\left(\frac{\lambda}{4 \pi \mathrm{d}_{1}}\right)^{2} \cdot \mathrm{PLF} .
$$

When we measure with three different antennas using identical setups characterized by $\left(h_{1}, h_{2}, R\right)$, shown in Fig. 1 , we obtain the following equations:

$$
\begin{aligned}
\left|\mathrm{S}_{21}\right|_{1 \rightarrow 2}^{2} & =\mathrm{G}_{1} \mathrm{G}_{2}\left(\frac{\lambda}{4 \pi \mathrm{d}_{1}}\right)^{2} \cdot \mathrm{PLF}_{1 \rightarrow 2} \\
\left|\mathrm{~S}_{21}\right|_{1 \rightarrow 3}^{2} & =\mathrm{G}_{1} \mathrm{G}_{3}\left(\frac{\lambda}{4 \pi \mathrm{d}_{1}}\right)^{2} \cdot \mathrm{PLF}_{1 \rightarrow 3} \\
\left|\mathrm{~S}_{21}\right|_{2 \rightarrow 3}^{2} & =\mathrm{G}_{2} \mathrm{G}_{3}\left(\frac{\lambda}{4 \pi \mathrm{d}_{1}}\right)^{2} \cdot \mathrm{PLF}_{2 \rightarrow 3}
\end{aligned}
$$

with $\mathrm{G}_{1}, \mathrm{G}_{2}, \mathrm{G}_{3}$ the realized gains of the three antennas. We position the antennas in such a way that the $\mathrm{PLF}_{\mathrm{i} \rightarrow \mathrm{j}}$ factors are 
approximated to one. Solving (6), (7), and (8), we obtain the realized gains.

Using the same notations as in formula (3), the power density $W$ at distance $R$ in the far field of the source antenna can be derived

$$
W=\frac{\mathrm{G}_{\mathrm{t}} \mathrm{P}_{\mathrm{i}}}{4 \pi R^{2}}=\frac{E^{2}}{120 \cdot \pi}
$$

$\mathrm{G}_{\mathrm{t}}$ is the realized gain of the transmitting antenna, $E$ is the electric field $(\mathrm{V} / \mathrm{m})$. Considering (1) and combining formula (9) with formula (3) also equal to $\mathrm{V}^{2} / 50$ (50 $\Omega$ measurement system), the antenna factor $\left[\mathrm{dB}\left(\mathrm{m}^{-1}\right)\right]$ is derived

$$
\mathrm{AF}=20 \cdot \log \left(\mathrm{f}_{\mathrm{M}}\right)-29.78-\mathrm{G}-10 \cdot \log (\mathrm{PLF})
$$

with $f_{M}$ the frequency in $\mathrm{MHz}$.

\section{Improvements}

Although we use an absorbing ground plane, destructive interference between the direct beam and the reflected beam could still appear, resulting in unreliable results. In Smith [1], [2], this problem is avoided by scanning the height of the receiving antenna until the maximum output voltage is measured. In our method, we split the considered frequency range in bands. In each frequency band, the distance between both antennas is changed after determining the path length difference $d$ between both beams. For horizontal polarization and using the notations of Fig. 1 we get $d=d_{2}-d_{1}$

$$
\mathrm{d}=\left[R^{2}+\left(h_{1}+h_{2}\right)^{2}\right]^{1 / 2}-\left[R^{2}+\left(h_{1}-h_{2}\right)^{2}\right]^{1 / 2} .
$$

If $d=\mathrm{n} \cdot \lambda$ with $\lambda$ the wavelength and $\mathrm{n}=1,2, \ldots$, then both beams will interfere destructively. Before measuring, we choose the height of the transmitting antenna $h_{1}$ and the height of the receiving antenna $h_{2}$. This choice is made such that the antennas are polarization matched (PLF is close to 1) and that the antennas are positioned high enough above the absorbing ground plane. This is because of the fact that the more orthogonal the beam hits the absorbers on the ground the better it will be absorbed. We then determine R, the distance between both antennas, in a frequency band using Fig. 4 and formula (11). To produce this figure, the reflection coefficient of the ground was approximated to one in amplitude and $180^{\circ}$ in phase. The points where destructive interference occurs are indicated with black spots. The frequencies in the neighborhood of these points of destructive interference should be avoided because at these nulls it will be impossible to solve (6)-(8) and the steep gradient of the field in the region of a null can result in large measurement errors due to small errors in antenna positioning [1], [2].

We have not yet taken into account residual reflections. To this end, we perform a de-embedding step. We describe two methods of de-embedding the reflected beam: The first one is performing an additional measurement by placing an absorber in between the two antennas. The second method uses the inverse fast Fourier transform (FFT) to obtain the time-domain signals and we then apply time-domain gating [12] and [13].

The de-embedding step by placing an absorber in between the two antennas is shown in Fig. 5. The absorption eliminates the direct beam. This results in the measurement of all the reflections by the surrounding environment, represented by $\mathrm{S}_{21}^{\text {reflected }}$.

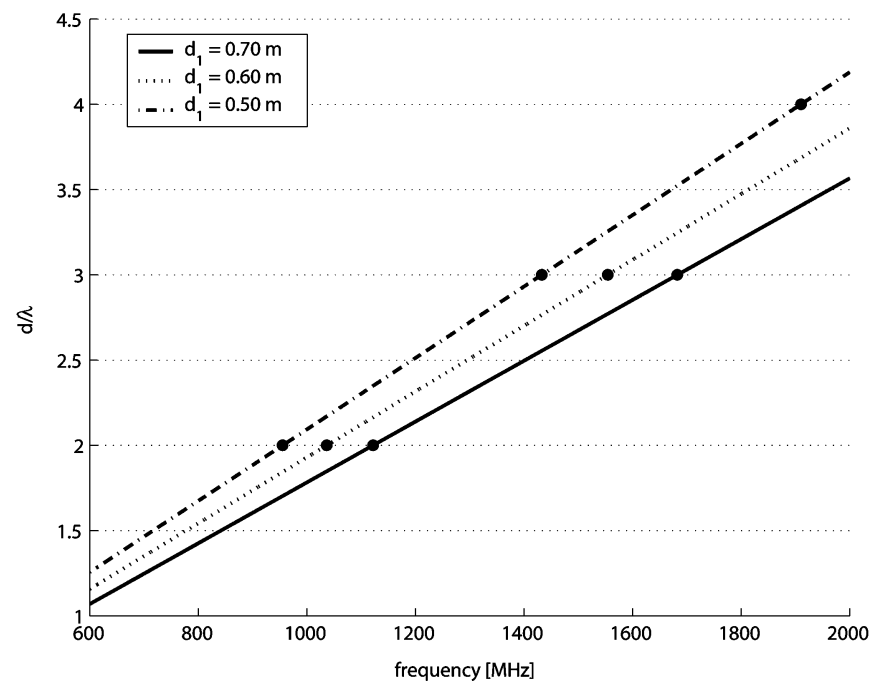

Fig. 4. Determination of $d / \lambda$ for three values of $d_{1}\left(h_{1}=0.60 \mathrm{~m}\right.$ and $h_{2}=0.45 \mathrm{~m}$ ). The black spots indicate the frequency points where destructive interference occurs.

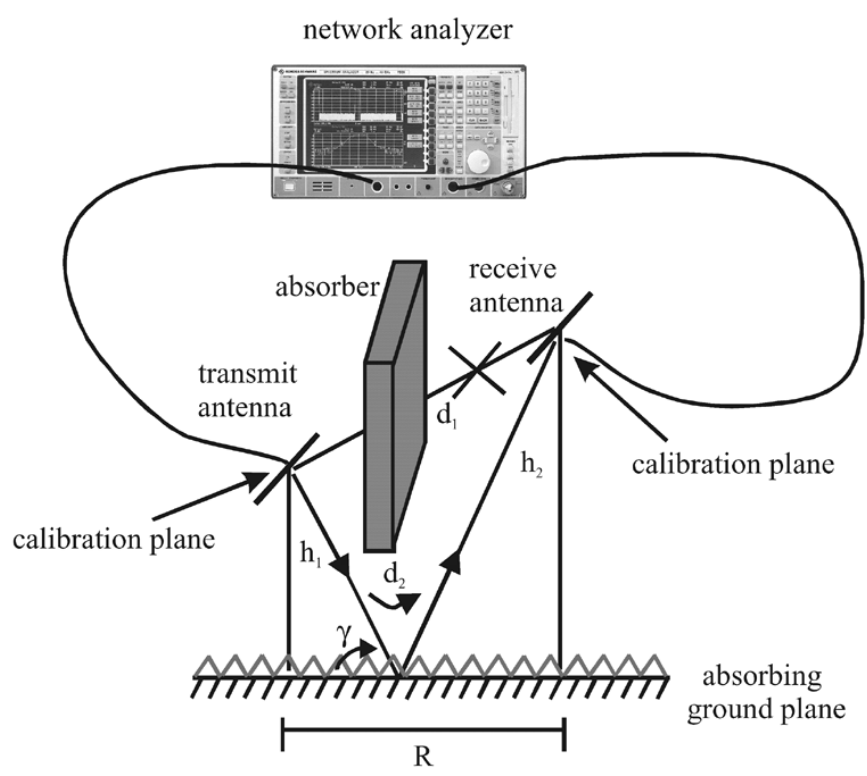

Fig. 5. De-embedding by placing an absorber in between the two antennas.

We take the reflections into account by subtracting $S_{21}^{\text {reflected }}$ from $S_{21}^{\text {meas }}$, where $S_{21}^{\text {meas }}$ represents the actual measurement

$$
\left|S_{21}^{\text {meas }}-S_{21}^{\text {reflected }}\right|^{2}=G_{\mathrm{t}} G_{\mathrm{r}}\left(\frac{\lambda}{4 \pi \mathrm{d}_{1}}\right)^{2} \cdot \mathrm{PLF}
$$

with $\mathrm{PLF} \approx 1$.

In the second de-embedding method, we first select the frequency range where the antenna factors must be determined. An appropriate configuration of the antennas is selected to avoid destructive interference in the desired frequency range. To obtain enough resolution in the time domain to distinguish the direct and reflected beam, a measurement is performed in a much larger frequency range. Using a tenth-order Butterworth bandpass filter, the $S_{21}(\mathrm{f})$-parameters in the desired frequency range are obtained. We then take the inverse FFT of the $\mathrm{S}_{21}$ (f)-parameters to obtain $\mathrm{S}_{21}(\mathrm{t})$. Next, we apply a time-domain gating technique [12], [13] to eliminate the residual reflection: The latest 


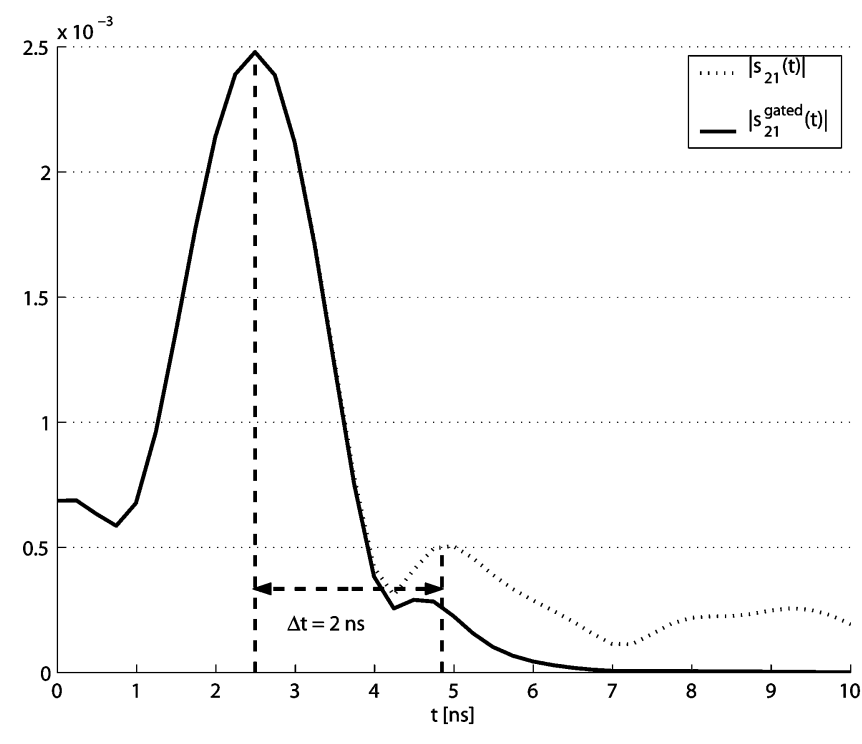

Fig. 6. Application of time-domain gating by filtering the reflected beam arriving 2 ns later than the direct beam.

arriving time-components (the reflected beam will arrive later than the direct beam) of $\mathrm{s}_{21}(\mathrm{t})$ are suppressed by a tenth-order Butterworth digital bandpass filter. This type of filter is selected because of its flat passband and the absence of side lobes. The difference of the propagation time between the direct and the reflected beam can be determined using formula (11). After filtering, we obtain $\mathrm{s}_{21}^{\text {gated }}(\mathrm{t})$. Finally we take the FFT to obtain $\mathrm{S}_{21}^{\text {gated }}(\mathrm{f})$ and use formula (13) to relate $\mathrm{S}_{21}^{\text {gated }}(\mathrm{f})$ to the gains

$$
\left|\mathrm{S}_{21}^{\text {gated }}(\mathrm{f})\right|^{2}=\mathrm{G}_{\mathrm{t}} \mathrm{G}_{\mathrm{r}}\left(\frac{\lambda}{4 \pi \mathrm{d}_{1}}\right)^{2} \cdot \mathrm{PLF}
$$

with $\mathrm{PLF} \approx 1$.

To further explain the de-embedding method based on the inverse FFT we show as an example the determination of the antenna factor of the conical dipole antenna in the frequency range $1100-1400 \mathrm{MHz}$. We used as heights $h_{1}=0.60 \mathrm{~m}$ and $h_{2}=0.45 \mathrm{~m}$. Using Fig. 4 and formula (11), the distance between both antennas is chosen in such a way that no destructive disturbance occurs in the considered frequency range. The distance between both antennas is $0.60 \mathrm{~m}$. For this configuration the difference of the propagation time between the direct and the reflected beam is $2 \mathrm{~ns}$. To obtain a resolution smaller than $2 \mathrm{~ns}$, it is necessary to use for the FFT a $\Delta \mathrm{f}>1 /(2 \mathrm{~ns})=500 \mathrm{MHz}$. The three measurements of each 801 points are performed from $300 \mathrm{kHz}$ to $4 \mathrm{GHz}$, therefore, $\Delta \mathrm{f}=3.9997 \mathrm{GHz}$ is large enough. Fig. 6 shows how we filtered the reflected beam in the time domain. The measurement from Fig. 6 is performed with the $15 \mathrm{~cm}$ dipole and the conical dipole antenna using the configuration described above. The results of both de-embedding methods and a comparison between both methods will be shown in Section III.

Finally, we had to take into account that the $15 \mathrm{~cm}$ dipole $(\lambda / 2$ at $900 \mathrm{MHz})$ antenna is almost insensitive for frequencies higher than $1600 \mathrm{MHz}$, while the $7.5 \mathrm{~cm}$ dipole $(\lambda / 2$ at 1800 $\mathrm{MHz})$ antenna becomes insensitive for frequencies lower than $800 \mathrm{MHz}$. So it is advisable to use the $15 \mathrm{~cm}$ dipole for the calibration from 600 to $1100 \mathrm{MHz}$ and the $7.5 \mathrm{~cm}$ dipole for the

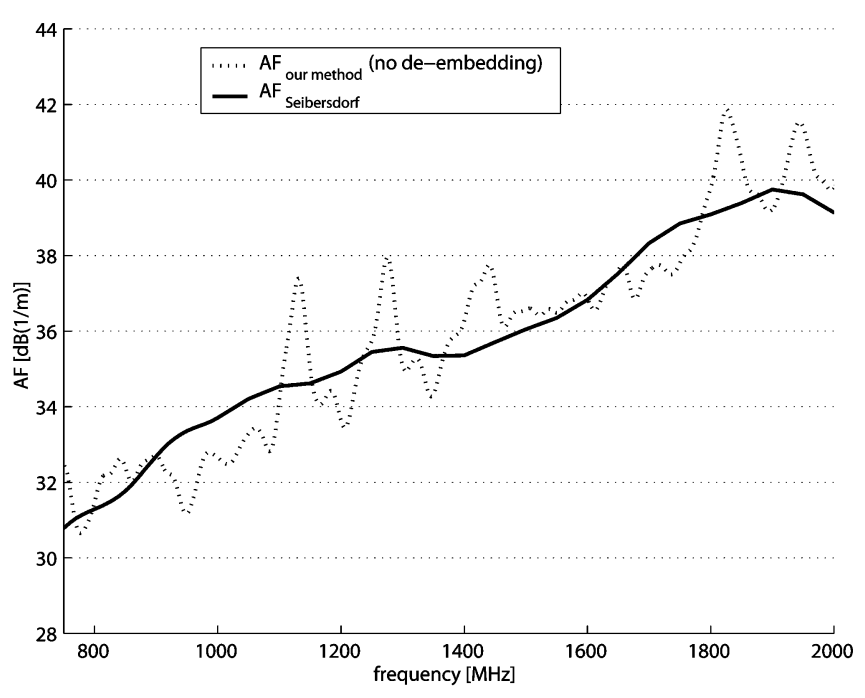

Fig. 7. Comparison of the antenna factors of the conical dipole antenna, respectively, obtained by the Austrian Research Center in Seibersdorf and obtained with our method without de-embedding.

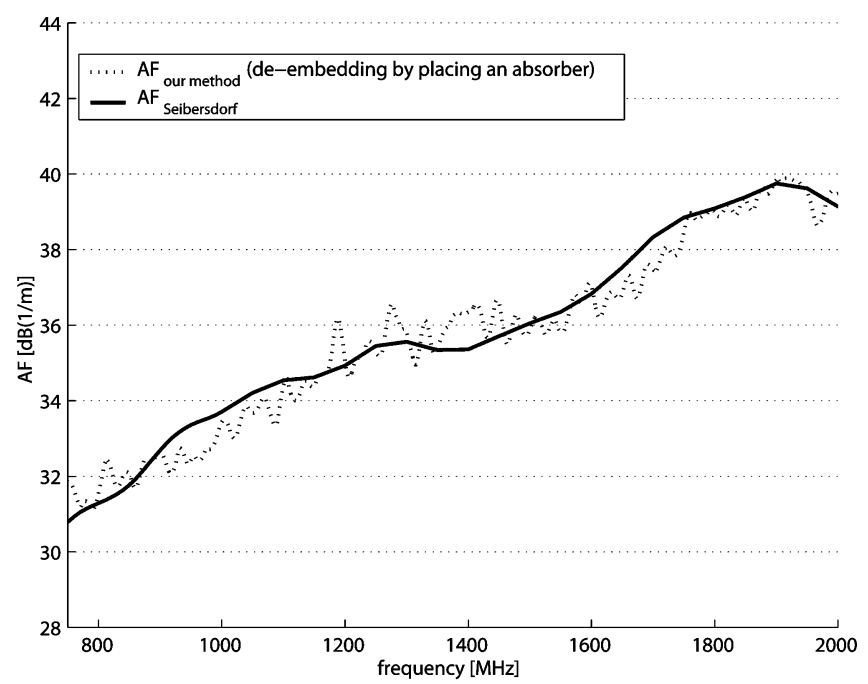

Fig. 8. Comparison of the antenna factors of the conical dipole antenna respectively, obtained by the Austrian Research Center in Seibersdorf and obtained with our method with de-embedding by placing an absorber between both antennas.

calibration from 1400 to $2000 \mathrm{MHz}$. In between those frequency ranges, we can use both dipoles.

\section{RESULTS}

To check the accuracy of our method, we compare the antenna factor of the conical dipole antenna, determined with our method, with the data obtained from the Austrian Research Center Seibersdorf where the antenna calibration is performed in an anechoic chamber. For our method, we used as heights $h_{1}=0.60 \mathrm{~m}$ and $h_{2}=0.45 \mathrm{~m}$. We show the results obtained without de-embedding and obtained with the two presented de-embedding methods in Figs. 7, 8, and 9. Table I lists the mean and maximum deviation. As can be noted there is already improvement by placing an absorber: The maximum deviation is reduced by $1.5 \mathrm{~dB}$. However, we obtain the best results with 


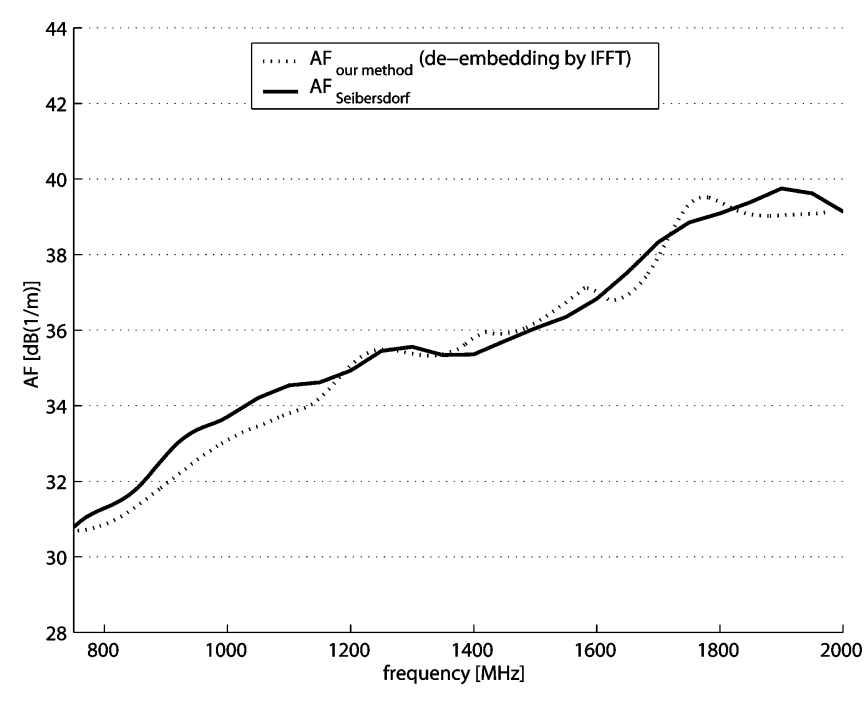

Fig. 9. Comparison of the antenna factors of the conical dipole antenna, respectively, obtained by the Austrian Research Center in Seibersdorf and obtained with our method with de-embedding by taking the inverse FFT.

TABLE I

COMPARISON OF THE ANTENNA FACTOR OF THE CONICAL DIPOLE ANTENNA ObTAINED by tHe AUSTRIAN RESEARCH CENTER IN SEIBERSDORF WiTH THE Result ObTained With OUR Method With AND Without De-EMBEDding

\begin{tabular}{c|c|c|c}
\hline $\begin{array}{c}\text { Deviation from antenna } \\
\text { factor obtained by the } \\
\begin{array}{c}\text { Austrian Research } \\
\text { Center Seibersdorf }\end{array}\end{array}$ & $\begin{array}{c}\text { Without de- } \\
\text { embedding } \\
{\left[\mathrm{dB}\left(\mathrm{m}^{-1}\right)\right]}\end{array}$ & $\begin{array}{c}\text { De-embedding by } \\
\text { placing an absorber } \\
{\left[\mathrm{dB}\left(\mathrm{m}^{-1}\right)\right]}\end{array}$ & $\begin{array}{c}\text { De-embedding by } \\
\text { taking the inverse } \\
\mathrm{FFT} \\
{\left[\mathrm{dB}\left(\mathrm{m}^{-1}\right)\right]}\end{array}$ \\
\hline Mean deviation & 0.67 & 0.41 & 0.39 \\
\hline Maximum deviation & 2.80 & 1.30 & 0.84 \\
\hline
\end{tabular}

the de-embedding by taking the inverse FFT: The deviation is maximal $0.84 \mathrm{~dB}$ and the average deviation is only $0.39 \mathrm{~dB}$. We can conclude that performing a de-embedding step largely improves the determination of the antenna factor. The Austrian Research Center Seibersdorf specified the antenna factor with an uncertainty of $\pm 1 \mathrm{~dB}$. So our result is lying within this uncertainty interval.

Putting an absorber in between the two antennas will introduce diffracted waves. This is a reason why the results of this method are worse than using the method with the inverse FFT. The size of the absorber we used is $60 \times 60 \times 3 \mathrm{~cm}^{3}$. To investigate the diffraction, we performed a worst-case finite difference time domain (FDTD) electromagnetic simulation with a perfectly conducting plate in between the two antennas and with a separation of $70 \mathrm{~cm}$ between the antennas. At $900 \mathrm{MHz}$ and with the dimensions of the plate equal to the absorber, we obtained that the electric fields at the receiving antenna due to diffraction are six times lower than the direct wave when no absorber is present. Using an absorber of dimensions $120 \times 120 \times 3 \mathrm{~cm}^{3}$ $(3.6 \lambda \times 3.6 \lambda \times 0.09 \lambda$ at $900 \mathrm{MHz})$ delivers diffracted fields that are more than 140 times lower. The method with an absorber in between the two antennas will deliver better results when using much larger absorbers, but this will be more expensive. The dimensions of the absorber are determined by the lowest frequency under consideration. Residual reflections from ceiling,

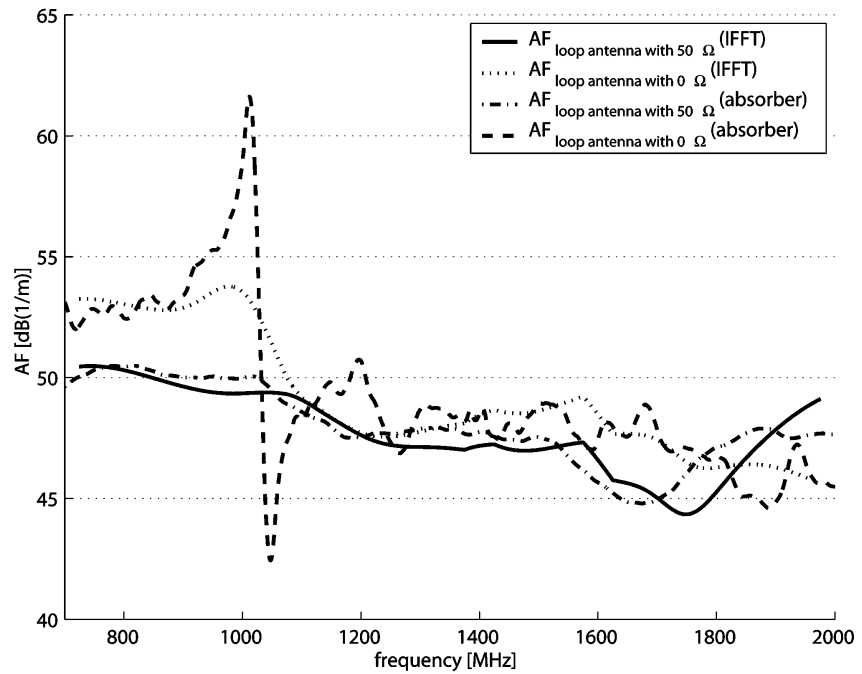

Fig. 10. Comparison of the antenna factor of the split-shield loop probes using both de-embedding methods.

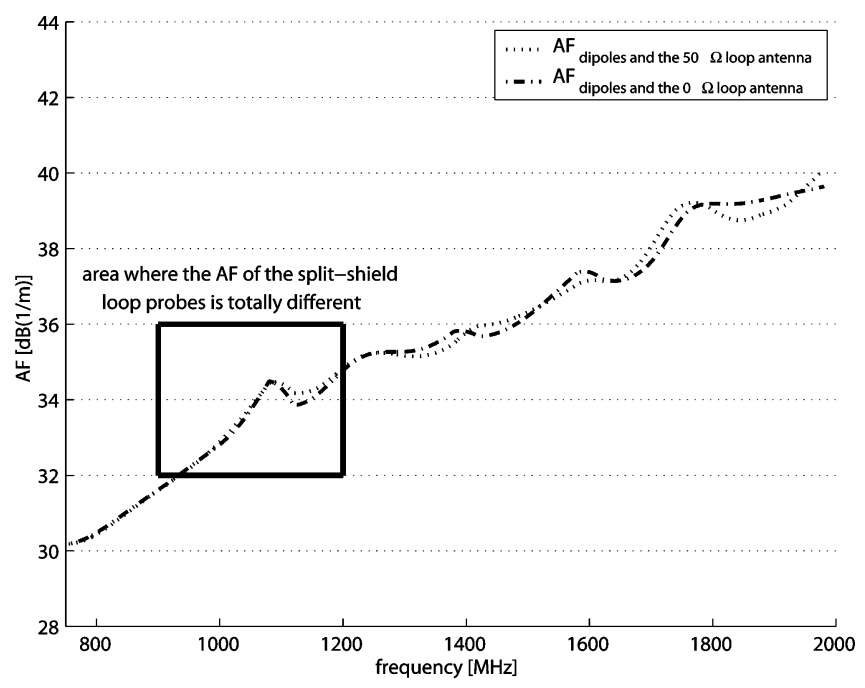

Fig. 11. Consistency check by comparison of the antenna factor of the conical dipole antenna using our three-antenna method with different antennas.

walls, ... will not be eliminated using this method in contrary to the method using the inverse FFT.

The antenna factors of the split-shield loop probes (used for measuring the magnetic field) determined by means of our method are shown in Fig. 10. For this figure, we used an absorbing ground plane and we performed the calibration for both de-embedding methods. A disadvantage of the $0 \Omega$ loop antenna is the appearance of the resonance peak at $1000 \mathrm{MHz}$. The resonance peak is smaller using the de-embedding by taking the inverse FFT because of the filtering in the time domain what comes down to a convolution and, thus, a smoothening in the frequency domain.

To check the consistency of our method, we performed our method with different antennas and we compared the antenna factor of the conical dipole antenna. We used the de-embedding by taking the inverse FFT. For the first measurement, the two other antennas were the two dipoles and the $0 \Omega$ split-shield loop antenna. For the second measurement, we used a $50 \Omega$ 
split-shield loop antenna in combination with the dipoles. In Fig. 11, we compare the obtained antenna factor of the conical dipole antenna. The deviation of the obtained antenna factors of the conical dipole antenna is very small, despite the fact that the antenna factor of the $0 \Omega$ split-shield loop antenna is totally different to the one of the $50 \Omega$ split-shield loop antenna in the frequency range of 900-1200 MHz. The average difference of both antenna factors for the whole frequency range is only $0.15 \mathrm{~dB}$; the maximum deviation is $0.49 \mathrm{~dB}$. This shows that our method is consistent.

\section{CONCLUSION}

We have presented an improved method to determine the antenna factor. From the results shown in this paper, we can conclude that our calibration method is accurate and consistent. By using antennas with totally different antenna factors, the mean deviation is only $0.15 \mathrm{~dB}$ and the maximum deviation is only $0.49 \mathrm{~dB}$ for the antenna factor of a conical dipole antenna. The use of an absorbing ground plane and performing a de-embedding step eliminate undesired reflections. Furthermore, the calibration is easy to perform with a network analyzer, and this method does not need to be performed in an anechoic chamber to be accurate, resulting in a low-cost method.

\section{REFERENCES}

[1] A. A. Smith JR., "Standard-site method for determining antenna factors," IEEE Trans. Electromag. Compat., vol. 24, no. 3, pp. 316-322, Aug. 1982.

[2] A. A. Smith JR, F. German, and B. Pate, "Calculation of site attenuation from antenna factors," IEEE Trans. Electromag. Compat., vol. 24, no. 3, pp. 301-316, Aug. 1982.

[3] American National Standard for Electromagnetic Compatibility-Radiated Emission Measurements in Electromagnetic Interference (EMI) Control-Calibration of Antennas (9 $\mathrm{kHz}$ to $40 \mathrm{GHz}$ ). ANSI C63.41998.

[4] T. Moroika and K. Komiyama, "Measurements of antenna characteristics above different conducting planes," IEEE Trans. Instrum. Meas., vol. 50, no. 2, pp. 393-396, Apr. 2001.

[5] R. McConnell, "A method of determining free space antenna factor on an open area test site," in IEEE Intl. Symp. Electromag. Compat., vol. 2, Washington, DC, Aug. 2000, pp. 499-504.

[6] Z. Chen and M. Foegelle, "An improved method for determining normalized site attenuation using log periodic dipole arrays," in IEEE Intl. Symp. Electromag. Compat., vol. 2, Washington, DC, Aug. 2000, pp. 511-516.
[7] A. Aykan, "Calibration of circular loop antennas," IEEE Trans. Instrum Meas., vol. 47, no. 2, pp. 446-452, Apr. 1998.

[8] J. Glimm, R. Harms, K. Münter, M. Spitzer, and R. Pape, "A singleantenna method for traceable antenna gain measurement," IEEE Trans. Electromag. Compat., vol. 41, no. 4, pp. 436-439, Nov. 1999.

[9] C. Carobbi, L. Millanta, and L. Chiosi, "The high-frequency behavior of the shield in the magnetic-field probes," in IEEE Intl. Symp. Electromag. Compat., vol. 1, Washington, DC, Aug. 2000, pp. 35-40.

[10] C. A. Balanis, Antenna Theory Analysis and Design. New York: Wiley, 1982, pp. 100-163.

[11] S. A. Schelkunoff and H. T. Friis, Antennas, Theory, and Practice. New York: Wiley, 1952.

[12] E. K. Miller, Time-Domain Measurements in Electromagnetics. New York: Van Nostrand, 1986, pp. 416-455.

[13] R. Yagüe, A. Ibars, and L. Martinez, "Analysis and reduction of the distortions induced by time-domain filtering techniques in network analyzers," IEEE Trans. Instrum. Meas., vol. 47, no. 4, pp. 930-934, Aug. 1998.

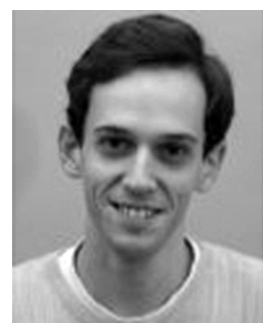

Wout Joseph was born in Ostend, Belgium, on October 21, 1977. He received the M.Sc. degree in electrical engineering from Ghent University, Ghent, Belgium, in 2000 and is currently pursuing the Ph.D. degree in the Department of Information Technology (INTEC), Ghent University.

Since September 2000, he has been involved in research on measuring and modeling of electromagnetic fields around base stations for mobile communications related to the health effects of the exposure to electromagnetic radiation. $\mathrm{He}$ is interested in electromagnetic field measurements, antennas, and calibration.

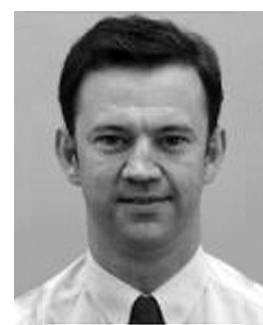

Luc Martens (M'92) was born in Gent, Belgium, on May 14, 1963. He received the M.Sc. degree in electrical engineering from Ghent University, Belgium, in July 1986.

From September 1986 to December 1990, he was a Research Assistant at the Department of Information Technology (INTEC), Ghent University. During this period, his scientific work was focused on the physical aspects of hyperthermic cancer therapy. His research work dealt with electromagnetic and thermal modeling and with the development of measurement systems for that application. This work led to a Ph.D. degree in December 1990. Since January 1991, he has been a member of the permanent staff of the Interuniversity MicroElectronics Centre (IMEC), Ghent, and is responsible for the research on experimental characterization of the physical layer of telecommunication systems at INTEC. His group also studies topics related to the health effects of wireless communication devices. Since April 1993, he has been a Professor in electrical applications of electromagnetism at Ghent University. 